\title{
Absorbing Boundary Conditions for the Numerical Simulation of Waves
}

\author{
By Bjorn Engquist* and Andrew Majda**
}

\begin{abstract}
In practical calculations, it is often essential to introduce artificial boundaries to limit the area of computation. Here we develop a systematic method for obtaining a hierarchy of local boundary conditions at these artificial boundaries. These boundary conditions not only guarantee stable difference approximations but also minimize the (unphysical) artificial reflections which occur at the boundaries.
\end{abstract}

Introduction. When calculating solutions to partial differential equations it is often essential to introduce artificial boundaries to limit the area of computation. Important areas of application which use artificial boundaries are local weather prediction (see [8] and [9]), geophysical calculations involving acoustic and elastic waves (see [6] and [7]), and a variety of other problems in fluid dynamics. One needs boundary conditions at these artificial boundaries in order to guarantee a unique and well-posed solution to the differential equation. In turn, this is a necessary condition to guarantee stable difference approximation. Of course, one hopes that these artificial boundaries and boundary conditions affect the solution in a manner such that it closely approximates the free space solution which exists in the absence of these boundaries. In particular, one would like to minimize the amplitudes of waves reflected from these artificial boundaries.

In this work we develop perfectly absorbing boundary conditions for general classes of wave equations by applying the recently developed theory for reflection of singularities for solutions of differential equations (see [2], [3] , [4]). Unfortunately, these boundary conditions necessarily have to be nonlocal in both space and time and thus are not useful for practical calculations. Hence, in the main part of the paper, we derive a hierarchy of highly absorbing local boundary conditions which approximate the theoretical nonlocal boundary condition. Of course, it is also very important for applications that these boundary conditions generate well-posed mixed initial boundary value problems. (See [1] for a discussion of well-posedness.) The general approach is applied specifically to produce absorbing artificial boundaries for the acoustic wave equation in Cartesian and polar coordinates and for the linearized shallow water equations in two space dimensions.

We illustrate our results by the following hierarchy of absorbing boundary conditions at the wall $x=0$ for the scalar wave equation,

$$
w_{t t}=w_{x x}+w_{y y}, \quad t, x \geqslant 0,
$$

Received November 23, 1976.

AMS (MOS) subject classifications (1970). Primary 65 M05, 65N99.

*Engquist's work was partially supported by S.E.P. from the Department of Applied Earth Sciences at Stanford University.

** Majda was partially supported by N.S.F. Grant MCS 76-10227. 


$$
\begin{gathered}
w_{x}-\left.w_{t}\right|_{x=0}=0, \\
w_{x t}-w_{t t}+1 /\left.2 w_{y y}\right|_{x=0}=0, \\
w_{x t t}-w_{t t t}-1 / 4 w_{x y y}+3 /\left.4 w_{t y y}\right|_{x=0}=0 .
\end{gathered}
$$

Each of these boundary conditions gives a well-posed mixed initial boundary value problem for the wave equation. We present an example in Section 1 where another obvious highly absorbing approximation generates a strongly ill-posed mixed problem. Thus, local absorbing approximations must be chosen carefully by using the normal mode analysis for mixed problems (see [1] for definitions) as a guideline. All of the above boundary conditions are perfectly absorbing at normal incidence. For a $45^{\circ}$ angle of incidence, (1), (2), and (3) reflect waves with amplitudes, respectively, 17\%, $3 \%$, and $.5 \%$ of the amplitude of the incident wave. Close to glancing, all reflection coefficients tend to unity. This is inherent in the problem; however, the corresponding reflected waves propagate slowly in the normal direction and hence do not affect the calculation in the interior.

In Section 1, we develop a theory of highly absorbing boundary conditions for second order wave equations. In Section 2, an analogous theory is developed for general symmetric hyperbolic systems. We illustrate this theory in Section 3 by explicitly calculating absorbing boundary conditions for the linearized shallow water equations; included in this section are some amusing observations from perturbation theory which have bearing here. Finally, in Section 4, mixed initial boundary problems with absorbing boundary conditions for the scalar wave equation and the linearized shallow water equations are approximated by difference schemes. The results of numerical experiments displayed here strongly indicate the value of our systematic approach as a practical tool.

The topic in this paper has been of considerable recent interest in the computational literature (see [9], [10], [11], [12], and [13]). In [9] and [11] the analogue of the first approximation (of Sommerfeld type) is implemented in nonlinear calculations while in [12] a special approximation is developed then analyzed in one space dimension. The wave equation in a half-space is studied in [10] and [13]. The starting point for [10] appears to be the same nonlocal perfectly absorbing condition developed in (1.6) below. The methods in [10] treat the nonlocal boundary condition directly and require storage of from three to six time levels on the boundary while in [13] solutions of the Neumann and Dirichlet problems are combined. The methods described in both of these papers are ingenious but rather special and appear to apply only to the constant coefficient scalar wave equation in a half-space. (In [13], some equations of elasticity are also analyzed.)

1. Highly Absorbing Boundary Conditions for Second Order Wave Equations. In this section we shall begin with a special example which motivates the manner in which we construct nonlocal perfectly absorbing boundary conditions together with suitable highly absorbing local approximations.

1.A. The Theoretical Nonlocal Boundary Condition. We consider solutions of 
the wave equation

$$
\square w=\frac{\partial^{2} w}{\partial t^{2}}-\frac{\partial^{2} w}{\partial x^{2}}-\frac{\partial^{2} w}{\partial y^{2}}=0
$$

in the half-space, $x \geqslant 0$. We denote the dual variables to $(y, t)$ by $(\omega, \xi)$. Special families of solutions to the wave equation representing waves travelling to the left are given by the plane waves

$$
w=e^{i\left(\sqrt{\xi^{2}-\omega^{2}} x+\xi t+\omega y\right)}
$$

with $\xi^{2}-\omega^{2}>0, \xi>0$. If $(\omega, \xi)$ is held fixed, one first order differential boundary condition which annihilates $w$ has the form,

$$
\left.\left(\frac{d}{d x}-i \sqrt{\xi^{2}-\omega^{2}}\right) w\right|_{x=0}=0 .
$$

Thus, for all waves as in (1.2) with $(\xi, \omega)$ held fixed, the boundary condition in (1.3) produces no reflections. On the other hand, more general wave packets travelling to the left can be represented by

$$
w(x, y, t)=\iint e^{i\left(\sqrt{\xi^{2}-\omega^{2}} x+\xi t+w y\right)} \rho(\xi, \omega) \hat{w}(0, \xi, \omega) d \xi d \omega .
$$

Here ^ denotes Fourier transform in the $(y, t)$ variables, $w(0, \xi, \omega)$ is a special amplitude function with support for large $(\xi, \omega)$ in the cone $\xi^{2}>\omega^{2}$, and $\rho(\xi, \omega)$ is a smooth function homogeneous of degree zero for $|\xi|+|\omega|$ large with support in $\left.\xi^{2}\right\rangle$ $\omega^{2}$ for $(\xi, \omega)$ large and identically one on a neighborhood of the support of $\hat{w}(0, \xi, \omega)$. We note that by Fourier's inversion formula, $w(0, y, t)$ is given by the inverse Fourier transform of the amplitude function, $\hat{w}(0, \xi, \omega)$. By superposition of the calculations for (1.3), the boundary condition which exactly annihilates singular wave packets travelling to the left of the form in (1.4) is given by

$$
\frac{d w}{d x}-\left.\iint e^{i(\xi t+\omega y)} i \sqrt{\xi^{2}-\omega^{2}} \rho \hat{w}(0, \xi, \omega) d \xi d \omega\right|_{x=0}=0 .
$$

Now, we express the mapping

$$
\iint e^{i(\xi t+\omega y)} i \sqrt{\xi^{2}-\omega^{2}} \rho \hat{w}(0, \xi, \omega) d \xi d \omega
$$

using the symbolic notation of pseudo-differential operators (see the article by Nirenberg in [3]). Suppose $b(y, t, \omega, \xi)$ is a formal asymptotic sum of terms with descending degrees of homogeneity in $(\xi, \omega)$ for $(\xi, \omega)$ large. Then a pseudo-differential operator is defined through the Fourier transform by the recipe,

$$
b\left(y, t, \frac{\partial}{\partial y}, \frac{\partial}{\partial t}\right) w=\iint e^{i(\omega y+\xi t)} b(y, t, i \omega, i \xi) \hat{w}(\xi, \omega) d \xi d \omega .
$$

Thus, the perfectly absorbing boundary condition in (1.5) can be written in the form

$$
\left.\left(\frac{d}{d x}-\rho\left(\frac{\partial}{\partial y}, \frac{\partial}{\partial t}\right) \sqrt{\frac{\partial^{2}}{\partial t^{2}}-\frac{\partial^{2}}{\partial y^{2}}}\right) w\right|_{x=0}=0 .
$$


Now, in general, it is not possible to design boundary conditions so that no reflections occur; nevertheless, it is possible to construct boundary conditions so that for an arbitrary highly singular wave packet (provided it contains no glancing directions) only low amplitude smooth reflections occur. (Of course, $\rho$ can be adjusted to exactly annihilate the wave packets from (1.4).) In fact, by applying the construction of Lax and Nirenberg from [3] and the algebraic criterion for perfect reflection developed by Majda and Osher in [2], one verifies that necessarily the only boundary conditions with these properties are multiples of the boundary condition in (1.6) with $\rho$ adjusted according to the largest angle of incidence of rays containing the singularities of $\omega$.

1.B. A Hierarchy of Highly Absorbing Local Boundary Conditions. Unfortunately, the perfectly absorbing boundary condition developed above in (1.6) is necessarily nonlocal in both space and time. This boundary condition is impractical from a computational point of view since to advance one time level at a single point requires information from all previous times over the entire boundary. Thus, we shall develop highly absorbing local approximations to the perfectly absorbing boundary condition in (1.6). Necessarily, we want to build boundary conditions satisfying the following two additional criteria:

(1) These boundary conditions are local.

(2) The boundary conditions lead to a well-posed mixed boundary value problem for the wave equation.

We necessarily need (2) to be satisfied; otherwise, it would be impossible to construct stable difference approximations to the associated boundary value problem while, as remarked above, (1) is essential for reasonable control of the operation count.

We take the symbol of the boundary condition from (1.6) given by

$$
d / d x-i \xi \sqrt{1-\omega^{2} / \xi^{2}}
$$

and approximate it at normal incidence $(\omega=0)$. Using the approximation $\sqrt{1-\omega^{2} / \xi^{2}}=1+O\left(\omega^{2} / \xi^{2}\right)$ and recalling that $i \xi$ corresponds to $\partial / \partial t$, we obtain

1st approximation:

$$
\left.\left(\frac{\partial}{\partial x}-\frac{\partial}{\partial t}\right) w\right|_{x=0}=0
$$

Using the next approximation (the first Taylor or Padé) to the square root, $\sqrt{1-\omega^{2} / \xi^{2}}=1-1 / 2 \omega^{2} / \xi^{2}+O\left(\omega^{4} / \xi^{4}\right)$ in (1.8) and multiplying by $i \xi$, we obtain a symbol

$$
i \xi \frac{\partial}{\partial x}+\xi^{2}-\frac{1}{2} \omega^{2}
$$

and this yields the boundary condition,

2nd approximation:

$$
\left.\left(\frac{\partial^{2}}{\partial x \partial t}-\frac{\partial^{2}}{\partial t^{2}}+\frac{1}{2} \frac{\partial^{2}}{\partial y^{2}}\right) w\right|_{x=0}=0
$$


Remark. The 1st approximation is a familiar one which works exactly for a single space dimension and could easily be derived from physical considerations. $\mathrm{Nu}$ merical experiments (see Section 4 of this paper) indicate that the 2nd approximation provides a significant improvement over the first (see the discussion below for the theoretical reasons); the authors do not know of any derivation of this approximation from "ad hoc" physical reasoning.

When $\xi=1, \omega$ has the interpretation of $\omega=\sin \theta$ where $\theta$ is the angle of incidence. Thus, if a wave of the form $a(\xi, \omega) e^{i \sqrt{\xi^{2}-\omega^{2}} x+i \omega y+i \xi t}$ strikes the boundary at the angle $\theta$, the first approximation produces a reflected wave

with amplitude $b$ given by

$$
b e^{-i \sqrt{\xi^{2}-\omega^{2}} x+i \omega y+i \xi t}
$$

$$
b=a\left(\frac{\cos \theta-1}{\cos \theta+1}\right),
$$

while the second approximation produces an even weaker reflected wave

with amplitude

$$
b e^{-i \sqrt{\xi^{2}-\omega^{2}} x+i \omega y+i \xi t}
$$

$$
b=-a\left|\frac{\cos \theta-1}{\cos \theta+1}\right|^{2} .
$$

We note that these conclusions are valid at both low and high frequencies (the numerical experiments in Section 4 confirm this).

We also claim that the 1 st and 2 nd approximation also satisfy the criterion in (2) above. Standard energy estimates prove that the 1st approximation is a maximal dissipative boundary condition and, therefore, is trivially well posed. The verification that the 2nd approximation is well posed is more subtle. We recall the general algebraic normal mode analysis for checking well-posedness (see the paper of Kreiss, [1]) specialized for the wave equation:

(1.11) A mixed initial boundary value problem, $B$, for the variable coefficient wave equation is well posed if there are no solutions to the frozen coefficient halfspace problems of the form

$$
w(s, \omega)=e^{-\sqrt{s^{2}+\omega^{2}} x+i \xi t+i \omega y}
$$

with $\operatorname{Res} \geqslant 0$ and satisfying,

$$
\square w(s, \omega)=0,\left.\quad B w(s, \omega)\right|_{x=0}=0 ;
$$

furthermore, for $|s|^{2}+|\omega|^{2}=1$ and $\operatorname{Res} \geqslant 0, B w(s, \omega)$ should satisfy $|B w(s, \omega)|$ $\geqslant c_{0}>0$.

Applying the criterion in (1.11) to the 2nd approximation, this approximation yields a well-posed problem unless

$$
-s \sqrt{s^{2}+\omega^{2}}=\left(s^{2}+1 / 2 \omega^{2}\right) \text { for some } s \text { with Res } \geqslant 0
$$

Squaring the identity in (1.12) we obtain that necessarily $\omega=0$ but when $\omega=0$ the above identity yields $2 s^{2}=0$, thus the problem is well posed.

It is natural to try higher order approximations to $\sqrt{1-\omega^{2} / \xi^{2}}$ for even better 
absorption. The 2 nd approximation involves approximating $\sqrt{1-\omega^{2} / \xi^{2}} \cong 1-$ $1 / 2 \omega^{2} / \xi^{2}$; this approximation coincides with both the first Taylor and first Padé approximations to $\sqrt{1+x}$ at $x=0$. Now, we investigate highly absorbing boundary conditions derived from the second Padé and Taylor approximations, respectively; these both reflect waves with amplitudes diminished by $|(\cos \theta-1) /(\cos \theta+1)|^{3}$ where $\theta$ is the angle of incidence.

We claim that the highly absorbing boundary condition derived from the $2 n d$ Padé approximation is well posed. To derive this boundary condition we use the second rational approximation

$$
\sqrt{1+x}=1+\frac{x}{2+x / 2}+O\left(|x|^{3}\right)
$$

and use a similar analysis as in the above to arrive at the third order boundary condition

\section{3rd approximation:}

$$
\left.\left(\frac{\partial^{3}}{\partial t^{2} \partial x}-\frac{1}{4} \frac{\partial^{3}}{\partial x \partial y^{2}}-\frac{\partial^{3}}{\partial t^{3}}+\frac{3}{4} \frac{\partial^{3}}{\partial t \partial y^{2}}\right) w\right|_{x=0}=0
$$

To check the well-posedness of this boundary value problem following (1.11) we need only show that there are no roots with Res $\geqslant 0$ for

$$
\left(s^{2}+\frac{\omega^{2}}{4}\right) \sqrt{s^{2}+\omega^{2}}+s^{3}+\frac{3}{4} s \omega^{2}=0 .
$$

If we set $s / \omega=z$, we obtain

$$
\left(z^{2}+1 / 4\right) \sqrt{1+z^{2}}=-\left(z^{3}+3 / 4 z\right)
$$

and squaring both sides and subtracting, we obtain $1 / 16=0$ thus there are no nontrivial solutions in (1.14) and the 3 rd approximation is well posed.

We also claim that in contrast to the above situation, the absorbing boundary condition corresponding to the second Taylor approximation is strongly ill posed. Thus, this local approximation is useless for numerical purposes. To derive this boundary condition we use

$$
\sqrt{1+x}=1+\frac{1}{2} x-\frac{1}{8} x^{2}+O\left(x^{3}\right)
$$

so that by following the above procedure we obtain the fourth order boundary condition,

4th approximation:

$$
\left.\left(\frac{\partial^{4}}{\partial x \partial t^{3}}-\frac{\partial^{4}}{\partial t^{4}}+\frac{1}{2} \frac{\partial^{4}}{\partial y^{2} \partial t^{2}}+\frac{1}{8} \frac{\partial^{4}}{\partial y^{4}}\right) w\right|_{x=0}=0
$$

To check the well-posedness of the above boundary condition, we must check that there are no roots with Res $\geqslant 0$ to

$$
-s \sqrt[3]{s^{2}+\omega^{2}}-s^{4}+\frac{1}{2} \omega^{2} s^{2}+\frac{1}{8} \omega^{4}=0 .
$$


Dividing by $\omega^{4}$ and setting $z=s / \omega$, we obtain

$$
-z \sqrt[3]{1+z^{2}}-z^{4}+\frac{1}{2} z^{2}+\frac{1}{8}=0
$$

Consider $z$ with $z$ real and $z \geqslant 0$. At $z=0$, the above expression has the value $1 / 8$, while as $z$ tends to $\infty$, the expression in (1.17) tends to $-\infty$. Thus a nontrivial positive real root exists and these boundary conditions are strongly ill posed.

Remark \#1. These two examples indicate that one must be careful in choosing the appropriate local approximation to the nonlocal boundary condition in order to guarantee stability.

Remark \#2. In the situation where the initial data or inhomogeneous forcing functions have support up to the boundary, one must be very careful in applying the above boundary conditions so that no additional discontinuities are introduced. (This difficulty exists for any mixed problem.) We illustrate this difficulty in the following example:

If we consider the free space solution of

$$
w_{t t}-w_{x x}=0,\left.\quad w\right|_{t=0}=0,\left.\quad w_{t}\right|_{t=0}=1,
$$

then $w \equiv t$. On the other hand, if we straightforwardly compute using homogeneous absorbing boundary conditions,

$$
\begin{gathered}
\tilde{w}_{t t}-\widetilde{w}_{x x}=0 \quad \text { for } x \geqslant 0, \\
\tilde{w}_{t}-\left.\widetilde{w}_{x}\right|_{x=0}=0, \quad \widetilde{w}_{t=0}=0,\left.\quad \tilde{w}_{t}\right|_{t=0}=1,
\end{gathered}
$$

we get a solution with discontinuity in the first derivative given by

$$
\begin{aligned}
\widetilde{w} & =1 / 2(x+t), & & t \geqslant x, \\
& =t, & & t \leqslant x .
\end{aligned}
$$

To control this difficulty we must make our initial and boundary data compatible and solve an inhomogeneous absorbing boundary value problem

$$
\begin{aligned}
& \widetilde{\widetilde{w}}_{t t}-\widetilde{\widetilde{w}}_{x x}=0, \quad x \geqslant 0, t \geqslant 0, \\
& \widetilde{\widetilde{w}}_{t}-\left.\widetilde{\widetilde{w}}_{x}\right|_{x=0}=g(t) \quad \text { for } t \geqslant 0, \\
& \widetilde{\tilde{w}}_{t=0}=0,\left.\quad \widetilde{\widetilde{w}}_{t}\right|_{t=0}=1,
\end{aligned}
$$

where $g(t)$ is a forcing function with $g(0)=\lim _{x \rightarrow 0^{+}} \partial \widetilde{\widetilde{w}} / \partial t-\partial \widetilde{\widetilde{w}} / \partial x=1$ (the limits in the last equation are calculated using the Cauchy data). No additional singular waves are produced as long as $g(0)$ satisfies the above condition and with $g(t) \equiv 1$, we recover $\widetilde{\widetilde{w}} \equiv t \equiv w$. (General compatibility conditions for hyperbolic mixed problems are discussed in [5].)

1.C. Modifications for Curved Boundaries and Variable Coefficient Wave Equations. Here we sketch how more sophisticated versions of the above arguments yield nonlocal perfectly absorbing and highly absorbing local boundary conditions for variable coefficient wave equations. The arguments we give below work for general variable coefficient wave equations. For the application and simplicity in exposition we 
consider wave equations of the form

$$
L w \equiv\left(\left(\frac{\partial^{2}}{\partial x^{2}}+g(x, y) \frac{\partial^{2}}{\partial y^{2}}+b(x, y) \frac{\partial}{\partial x}\right)-\frac{\partial^{2}}{\partial t^{2}}\right) w=0
$$

with $g(x, y)>c_{0}>0$ in the half-space $x>0$; and we seek perfectly absorbing boundary conditions on the wall, $x=0$. The key for the constant coefficient wave equation was that it could be factored (within a smooth error) by using pseudo-differential operators into

$$
\square \cong\left(\frac{\partial}{\partial x}+\rho \sqrt{\frac{\partial^{2}}{\partial t^{2}}-\frac{\partial^{2}}{\partial y^{2}}}\right)\left(\frac{\partial}{\partial x}-\rho \sqrt{\frac{\partial^{2}}{\partial t^{2}}-\frac{\partial^{2}}{\partial y^{2}}}\right)
$$

near the relevant rays. Here $\cong$ denotes "within a smooth error". The factor $\partial / \partial x-$ $\rho \sqrt{\partial^{2} / \partial t^{2}-\partial^{2} / \partial y^{2}}$ corresponds to propagation to the left, while $\partial / \partial x+$ $\rho \sqrt{\partial^{2} / \partial t^{2}-\partial^{2} / \partial y^{2}}$ corresponds to propagation of reflected waves travelling to the right. To minimize the amplitude of reflected waves travelling to the right, we chose the boundary condition in (1.6). For variable coefficient problems as in (1.18), the theory of pseudo-differential operators allows us to factorize general wave equations in the fashion as in (1.19). From a very special case of Lemma 1 on page 27 of the lectures by Nirenberg in [3], we conclude that the operator $L$ in (1.18) can be factored in the form,

$$
L \cong\left(\frac{\partial}{\partial x}+\lambda_{+}\left(x, y, \frac{\partial}{\partial y}, \frac{\partial}{\partial t}\right)\right)\left(\frac{\partial}{\partial x}-\lambda_{-}\left(x, y, \frac{\partial}{\partial y}, \frac{\partial}{\partial t}\right)\right),
$$

where $\lambda_{-}$is a pseudo-differential operator with a symbol which has the formal asymptotic expansion,

$$
\lambda_{-}(x, y, \omega, \xi) \cong \lambda_{-}^{1}(x, y, \omega, \xi)+\lambda_{-}^{0}(x, y, \omega, \xi)+\lambda_{-}^{-1}(x, y, \omega, \xi)++,
$$

where $\lambda^{j}$ is a homogeneous function 'of degree $j$. By using Theorems 1 and 2 of the work of Majda and Osher in [2], we verify that the perfectly absorbing boundary condition is given by

$$
\left.\left(\frac{\partial}{\partial x}-\lambda_{-}\left(x, y, \frac{\partial}{\partial y}, \frac{\partial}{\partial x}\right)\right) w\right|_{x=0}=0
$$

The coefficients, $\lambda_{-}^{j}$, are recursively determined by the composition formulas of pseudo-differential operators as in [3]. One calculates that the first coefficient has symbol given by

$$
\lambda_{-}^{1}(x, y, \xi, \omega)=i \sqrt{\xi^{2}-g(x, y) \omega^{2}} .
$$

The second coefficient is determined recursively from the first to be

$$
\lambda_{-}^{0}(x, y, \xi, \omega)=\frac{b}{2}+\frac{1}{4} \frac{\partial g \omega^{2} / \partial x}{\left(\xi^{2}-g(x, y) \omega^{2}\right)}+\frac{i g(x, y) \partial g \omega^{3} / \partial y}{2\left(\xi^{2}-g(x, y) \omega^{2}\right)^{3 / 2}} .
$$


To make suitable local approximations, for simplicity we retain the first two terms $\lambda_{-} \cong \lambda_{-}^{1}+\lambda_{-}^{0}$ in the asymptotic expansion of $\lambda_{-}$(retaining more terms clearly improves the method but is too cumbersome to display here). Thus, we must approximate the nonlocal boundary condition,

$$
\begin{aligned}
\frac{\partial}{\partial x}-\left(\sqrt{\frac{\partial^{2}}{\partial t^{2}}-g(x, y) \frac{\partial^{2}}{\partial y^{2}}}+\frac{b}{2}\right. & -\frac{1}{4} \frac{(\partial g / \partial x)\left(\partial^{2} / \partial y^{2}\right)}{\left(\partial^{2} / \partial t^{2}-g(x, y) \partial^{2} / \partial y^{2}\right)} \\
& \left.+\frac{g(x, y) \partial g / \partial y}{\left(\partial^{2} / \partial t^{2}-g(x, y) \partial^{2} / \partial y^{2}\right)^{3 / 2}}\right)\left.w\right|_{x=0}=0 .
\end{aligned}
$$

If we expand the symbols at normal incidence suing $\xi^{2}-g(x, y) \omega^{2} \cong \xi^{2}$, we obtain the first local approximation

$$
\left.\left(\frac{\partial}{\partial x}-\frac{\partial}{\partial t}-\frac{b}{2}\right) w\right|_{x=0}=0 .
$$

This approximation diminishes the amplitude of reflected waves by $O\left(|\omega / \xi|^{2}\right)$. Using $\left(1-\omega^{2} / \xi^{2}\right)^{1 / 2}=1-1 / 2 \omega^{2} / \xi^{2}$, etc., we obtain the second local approximation given symbolically by

$$
\frac{\partial}{\partial x}-i \xi\left(1-\frac{1}{2} g \frac{\omega^{2}}{\xi^{2}}\right)+\frac{b}{2}-\frac{1}{4} \frac{\partial g}{\partial x} \frac{\omega^{2}}{\xi^{2}} .
$$

We note that the difference between (1.25) and the symbol of $(1.23)$ is $O\left(|\omega / \xi|^{3}\right)$. Multiplying (1.25) by $\xi^{2}$, we obtain the second local approximation,

$$
\left.\left(\frac{\partial^{3}}{\partial x \partial t^{2}}-\frac{\partial^{3}}{\partial t^{3}}+\frac{1}{2} g \frac{\partial^{3}}{\partial t \partial y^{2}}+\frac{b}{2} \frac{\partial^{2}}{\partial t^{2}}+\frac{1}{4} \frac{\partial g}{\partial x} \frac{\partial^{2}}{\partial y^{2}}\right) w\right|_{x=0}=0
$$

By freezing coefficients and applying the identical calculations from the 1st and 2nd approximation in (1.9), (1.10) above in the normal mode analysis from (1.11), we conclude that the absorbing approximations in (1.24) and (1.26) yield well-posed problems.

The Special Case of Polar Coordinates. We merely specialize the above formula to polar coordinates in two dimensions where we construct highly absorbing boundary conditions on the circle of radius $a$. Here $L$ has the form

$$
L=\frac{\partial^{2}}{\partial r^{2}}+\frac{1}{r^{2}} \frac{\partial^{2}}{\partial^{2} \theta}+\frac{1}{r} \frac{\partial}{\partial r}-\frac{\partial^{2}}{\partial t^{2}}
$$

where $\partial / \partial r, \partial / \partial \theta$ correspond to $\partial / \partial x, \partial / \partial y$ in the above formulas and $b=1 / r, g=$ $1 / r^{2}$

Using (1.24), the first highly absorbing approximation on $r=a$ for the region $r \leqslant a$ is given by

$$
\left.\left(\frac{\partial}{\partial r}+\frac{\partial}{\partial t}+\frac{1}{2 a}\right) w\right|_{r=a}=0 .
$$

Using (1.26), the second highly absorbing approximation on $r=a$ is given by 


$$
\left.\left(\frac{\partial^{3}}{\partial r \partial t^{2}}+\frac{\partial^{3}}{\partial t^{3}}-\frac{1}{2 a^{2}} \frac{\partial^{3}}{\partial t \partial^{2} \theta}+\frac{1}{2 a} \frac{\partial^{2}}{\partial t^{2}}+\frac{1}{2} \frac{1}{a^{3}} \frac{\partial^{2}}{\partial \theta^{2}}\right) w\right|_{r=a}=0
$$

Remark. For $r=a$ with $r \longrightarrow \infty$, outgoing waves have the form,

$$
\frac{f(r-t)}{r^{1 / 2}}\left(a(\theta)+O\left(\frac{1}{r}\right)\right) \text {. }
$$

We observe that the first approximation from (1.27) precisely annihilates $f(r-t) a(\theta) / r^{1 / 2}$ on $r=a$. The approximation from (1.28) also compensates for angular dependence,i.e., the wave is not quite a spherical wave.

2. Highly Absorbing Boundary Conditions for First Order Symmetric Systems. We consider general variable coefficient first order strictly hyperbolic systems of the form

$$
\frac{\partial u}{\partial t}=\left(A_{1}(x, y) \frac{\partial}{\partial x}+A_{2}(x, y) \frac{\partial}{\partial y}+B(x, y)\right) u
$$

in the region $x \geqslant 0$ where $A_{1}, A_{2}$ are symmetric $m \times m$ matrices (general symmetric systems in regions with curved boundary, at least locally, can be mapped to a halfspace where they assume the form in (2.1)). Our objective is to design highly absorbing local boundary conditions for the system in (2.1). We assume that $A_{1}$ has the form

$$
A_{1}=\left(\begin{array}{ll}
A_{1}^{1} & 0 \\
0 & A_{1}^{2}
\end{array}\right)
$$

where

$$
A_{1}^{1}=\left(\begin{array}{ll}
\lambda_{1} & 0 \\
0 & \cdot \\
0 & \lambda_{k}
\end{array}\right)
$$

with $\lambda_{i}(x, y)<0$ and

$$
A_{1}^{2}=\left(\begin{array}{ll}
\lambda_{k+1} & 0 \\
0 & \cdot \lambda_{n}
\end{array}\right)
$$

with $\lambda_{k+j}>0$. As a consequence of strict hyperbolicity, $\lambda_{i} \neq \lambda_{j}$ for $i \neq j$.

2.A. The Theoretical Nonlocal Perfectly Absorbing Boundary Condition. We rewrite the equation in (2.1) as

$$
\frac{\partial u}{\partial x}=A \frac{\partial u}{\partial t}+E \frac{\partial u}{\partial y}+\widetilde{B u}
$$

where

$$
A=A_{1}^{-1}=\left(\begin{array}{ll}
\Lambda_{1} & 0 \\
0 & \Lambda_{2}
\end{array}\right)
$$


with $\Lambda_{1}<0$ and $\Lambda_{2}>0, E=-A_{1}^{-1} A_{2}, \widetilde{B}=-A_{1}^{-1} B$ and we define $M(\xi, \omega)$ by

$$
M(\xi, \omega) \equiv A i \xi+E i \omega .
$$

The theoretical construction to be used here parallels Nirenberg's factorization from part C of Section 1 and was developed by M. E. Taylor in [4] for general first order systems. More precisely, there exists a smooth matrix $V(\xi, \omega, x, y)$ invertible for all $(\xi, \omega)$ satisfying $|\omega| /|\xi|+|\omega|<c_{0}$ for some choice of $c_{0}$ and defining the symbol of a pseudo-differential operator so that if

$$
w=V\left(\frac{\partial}{\partial t}, \frac{\partial}{\partial y}, x, y\right) u,
$$

the equations in (2.3) assume the form

$$
\frac{\partial w}{\partial x}=\left(\begin{array}{cc}
\Lambda_{11}\left(\frac{\partial}{\partial t}, \frac{\partial}{\partial y}, x, y\right) & 0 \\
\Lambda_{21}\left(\frac{\partial}{\partial t}, \frac{\partial}{\partial y}, x, y\right) & \Lambda_{22}\left(\frac{\partial}{\partial y}, \frac{\partial}{\partial t}, x, y\right)
\end{array}\right) w
$$

where $\Lambda_{11}(\partial / \partial t, \partial / \partial y, x, y)$ is a $k \times k$ pseudo-differential operator of order 1 with principal symbol, $\Lambda_{11}$, evaluated at $(0,1, x, y)$, given by

$$
\Lambda_{11}(0,1, x, y)=\Lambda_{1}=\left(\begin{array}{ccc}
\lambda_{1}^{-1} & & 0 \\
0 & & \lambda_{k}^{-1}
\end{array}\right)
$$

and satisfying $\Lambda_{11}<0$ for $(\xi, \omega)$ with $|\omega| /|\xi|+|\omega|<c_{0}$. Here $\Lambda_{21}, \Lambda_{22}$ are $(m-k) \times k, m-k \times m-k$ matrix pseudo-differential operators of order 1 , respectively.

Once the differential equation has the form in (2.4) it is easy to write down the theoretical perfectly absorbing boundary condition at least for all waves striking at angles sufficiently close to normal incidence. Recall that $\Lambda_{11}^{1}$ has all the negative real eigenvalues corresponding to light rays flowing inward into $x>0$ for positive time (the doubting reader should consider the case of a single space dimension). Thus, the theoretical absorbing boundary condition annihilates these components for $x=0$. Let $\pi_{k}\left(w_{1}, \ldots, w_{k}, w_{k+1}, \ldots, w\right) \equiv\left(w_{1}, \ldots, w_{k}\right)$; the perfectly absorbing boundary condition is given by

$$
\left.\pi_{k} w\right|_{x=0}=\left.\pi_{k} V u\right|_{x=0}=0 .
$$

To gain some insight into the nature of this nonlocal condition, we recall Taylor's construction of $V(\xi, \omega, x, y)$ (see Section 1 of [4]).

$$
\begin{aligned}
V(\xi, \omega, x, y) \cong & V_{0}(\xi, \omega, x, y)+\xi^{-1} V_{-1}(\xi, \omega, x, y) \\
& +\xi^{-2} V_{-2}(\xi, \omega, x, y)++
\end{aligned}
$$

where each $V_{-j}$ is homogeneous of degree zero and $\cong$ is the asymptotic sense of pseudo-differential operators. One consequence of strict hyperbolicity is that $M(1,0)$ 
is a diagonal matrix with distinct entries; therefore, there exists a constant $c_{0}$ so that for $|\omega| /|\xi|+|\omega| \leqslant c_{0}, M(\xi, \omega)$ also has distinct eigenvalues; and we choose $V(\xi, \omega, x, y)$ to be a smoothly varying matrix, homogeneous of degree zero, so that $M(\xi, \omega)$ becomes block lower triangular, i.e.

$$
\left(\begin{array}{cc}
{ }_{1} \Lambda_{11} & 0 \\
{ }_{1} \Lambda_{21} & { }_{1} \Lambda_{22}
\end{array}\right) \equiv V_{0} M V_{0}^{-1}
$$

It is obvious that $M(\xi, \omega)$ has distinct eigenvalues, in general, only in a conic neighborhood of normal incidence $(\omega=0)$ (since at a value of $(\xi, \omega)$ corresponding to glancing for one of the sound speeds, two real roots coalesce and then become complex); and the constraint $|\omega| /|\xi|+|\omega| \leqslant c_{0}$ restricts the value of $\omega$ to the region where these eigenvalues stay distinct. We set $w_{0}=V_{0} u$, then $w_{0}$ formally satisfies the equation in (2.4) up to error terms which at the symbol level are bounded in $\xi$. We write $w_{1}=\left(1+K_{1}\right) w_{0}$ with $K_{1} V_{0}=\xi^{-1} V_{-1}$ and attempt to write an equation for $w_{1}$ in the form of (2.4) at least up to terms of order $\xi^{-1}$ at the symbol level. Using the composition formulas for pseudo-differential operators, we obtain that

$$
\begin{aligned}
& \left(1+K_{1}\right) V_{0}(M(\xi, \omega)+\widetilde{B}) V_{0}^{-1}\left(1+K_{1}\right)^{-1} \\
& =K_{1} V_{0} M(\xi, \omega) V_{0}^{-1} 0 V_{0} M(\xi, \omega) V_{0}^{-1} K_{1}+V_{0}\left(\widetilde{B}-\partial_{x} V_{0}\right) V_{0}^{-1}+O\left(\xi^{-1}\right) .
\end{aligned}
$$

We define $\widetilde{C} \equiv \widetilde{B}-\partial_{x} V_{0}$. From (2.9) the terms in the upper right-hand corner of (2.4) which are bounded in $\xi$ will vanish if we choose $K_{1}$ to have the form

$$
K_{1}=\left(\begin{array}{cc}
0 & \widetilde{K} \\
0 & 0
\end{array}\right),
$$

where $\widetilde{K}$ is an $k \times m-k$ matrix satisfying

$$
\widetilde{K}_{11} \Lambda_{22}-{ }_{1} \Lambda_{11} K_{1}+\widetilde{C}_{12}=0 \text {. }
$$

As was proved in Section 1 of [4], a unique choice of $\widetilde{K}_{1}$ satisfying the equations in (2.11) exists provided that

$$
{ }_{1} \Lambda_{22} \equiv \Lambda_{2} \quad \text { and } \quad{ }_{1} \Lambda_{11} \equiv \Lambda_{1}
$$

have disjoint spectra. The condition in (2.12) is satisfied here because the eigenvalues of $\Lambda_{2}$ are positive and those of $\Lambda_{1}$ are negative.

2.B. Highly Absorbing Local Approximations. To construct highly absorbing local approximations, we approximate the matrix $V(\xi, \omega, x, y)$ at normal incidence. Recall the asymptotic representation for $V$ from $(2.7)$. We take each $V_{-j}(\xi, \omega)$ and use Taylor's theorem to write

$$
\begin{aligned}
V_{-j}(\xi, \omega) & =V_{-j}\left(1, \frac{\omega}{\xi}\right) \\
& =V_{-j}(1,0)+\sum_{K=1}^{l}\left(\frac{\omega}{\xi}\right)^{K} \frac{1}{K !} \frac{\partial^{K}}{\partial \omega^{K}} V_{-j}(1,0)+O\left(\left|\frac{\omega}{\xi}\right|^{l+1}\right) .
\end{aligned}
$$


Using (2.7) and (2.13) in the theoretical boundary condition in (2.6) and retaining only terms with an error $O(|\omega / \xi|+|1 / \xi|)$, we obtain the first approximation,

1st approximation:

$$
\left.\pi_{k} V_{0} u\right|_{x=0}=0
$$

Looking back at (2.3), we see that this is merely the projection on the inflowing characteristics for positive time for the one-dimensional equation derived from (2.1) by setting $A_{2} \equiv 0$. A simple energy estimate shows that this boundary condition is maximal dissipative and is always well posed for (2.1). We remark that if the 1-dimensional wave equation was written as a first order system using $\left(u_{t}, u_{x}\right)$ as components, the boundary condition in (2.14) would coincide with the 1 st approximation from Section 1.

To construct the second approximation with reflected error $O\left(|\omega / \xi|^{2}+1 /|\xi|^{2}\right)$, we retain terms from $V_{0}$ up to order 1 and terms from $V_{-1}$ up to order zero to get the symbol

$$
\pi_{K}\left(V_{0}(1,0)+\frac{\omega}{\xi} \frac{\partial}{\partial \omega} V_{0}(1,0)+\frac{1}{\xi} V_{-}(1,0)\right) .
$$

Multiplying by $\xi$ we arrive at the second highly absorbing approximation,

2nd approximation:

$$
\left.\pi_{k}\left(\frac{d}{d t} V_{0}(1,0)+\frac{\partial}{\partial \omega} V_{0}(1,0) \frac{\partial}{\partial y}+V_{-1}(1,0)\right) u\right|_{x=0}=0
$$

where $V_{-1}$ is determined by the lower order term $B$ and the variable nature of the coefficients according to (2.9). We make the following conjecture:

The 2nd approximation always yields a well-posed boundary value problem for (2.1).

It is worthwhile mentioning that a highly absorbing approximation intermediate between the first and second approximations may be useful in situations where the effect of the lower order term $B$ is rather large. We have with reflected error $O\left(|\omega / \xi|+1 / \xi^{2}\right)$, the $1 \frac{112}{2}$ order approximation.

$$
\left.\pi_{k}\left(\frac{d}{d t} V_{0}(1,0)+V_{-1}(1,0)\right) \mu\right|_{x=0}=0
$$

This approximation is always well posed because its principal symbol is the same as the one in (2.14).

To complete the pattern, we retain the terms in (2.13) from $V_{-j}$ up to order $l-j$ and then multiply the symbol by $\xi^{l}$ to obtain the $l+1$ th highly absorbing approximation with reflected error $O\left(|\omega / \xi|^{l+1}+1 /|\xi|^{l+1}\right)$.

$l+1$ th approximation:

$$
\left.\pi_{K}\left(\sum_{j=0}^{l} \sum_{K=0}^{l-j} \frac{\partial^{l-j-K}}{\partial t^{l-j-K}} \frac{1}{K !} \frac{\partial^{K}}{\partial \omega^{K}} V_{-j}(1,0) \frac{\partial^{K}}{\partial y^{K}}\right) u\right|_{x=0}=0 .
$$

In view of the instability of the 4th approximation from Section 1 for the wave equa- 
tion, it is doubtful that in general for $l>1$ these boundary conditions would yield well-posed problems. Perhaps, using other approximations analogous to the Padé approximation from Section 1 would be useful here.

2.C. Some Perturbation Calculations Applied to Highly Absorbing Approxitions. In order to use the 2nd approximation from (2.15) in explicit problems, we need to evaluate $\partial V_{0}(1,0) / \partial \omega$; as we shall see below, this reduces to a standard calculation in perturbation theory. Set $\omega / \xi=\delta$ and write

$$
M\left(1, \frac{\omega}{\xi}\right)=\left(\begin{array}{ll}
\Lambda_{1} & 0 \\
0 & \Lambda_{2}
\end{array}\right)+\delta\left(\begin{array}{ll}
E_{11} & E_{12} \\
E_{21} & E_{22}
\end{array}\right)
$$

Recall that $V_{0}$ is chosen to guarantee the relation in (2.8). We write $V_{0}$ as $V_{0}=$ $I+\delta_{1} V_{0}+O\left(\delta^{2}\right)$, then it follows that

$$
\frac{\partial V_{0}}{\partial \omega}(1,0)={ }_{1} V_{0}
$$

To calculate ${ }_{1} V_{0}$ is a standard problem (identical to the one studied in [4] and (2.9), (2.10), (2.11) above). Substituting $V_{0}=I+\delta_{1} V_{0}+O\left(\delta^{2}\right)$ and requiring the upper right block of $V_{0}\left(A_{1}+\delta E\right) V_{0}^{-1}$ to vanish to order $O\left(\delta^{2}\right)$, we obtain the equation

$$
X \Lambda_{2}-\Lambda_{1} X+E_{12}=0
$$

where we choose ${ }_{1} V_{0}$ of the form

$$
{ }_{1} V_{0}=\left(\begin{array}{ll}
0 & X \\
0 & 0
\end{array}\right)
$$

Following (2.9), (2.10), (2.11), $X$ is uniquely determined by solving the linear equations in (2.20); thus, $\partial V_{0}(1,0) / \partial \omega$ is determined.

We shall now develop a special perturbation lemma under additional hypotheses.

These hypotheses concerning the $k \times(m-k)$ matrix, $X$, from $(2.20)$ are that

$$
k \geqslant m-k, \quad X \text { has rank } m-k \text {. }
$$

In this situation, we prove that by choosing $V_{0}=S+O\left(\delta^{3}\right)$ through the linear change of variables $S \equiv I+\delta R$ with $R$ appropriately chosen, we have

$$
S(A+\delta E) S^{-1}=\left(\begin{array}{ll}
\Lambda_{11} & O\left(\delta^{3}\right) \\
\Lambda_{21} & \Lambda_{22}
\end{array}\right)
$$

Thus, under these hypotheses, we have the special 2 nd approximation,

2nd approximation*:

$$
\left.\pi_{k}\left(\frac{d}{d t} V_{0}(1,0)+R \frac{\partial}{\partial y}+V_{-1}(1,0)\right) u\right|_{x=0}=0
$$

If for simplicity $A_{1}, A_{2}$ have constant coefficients, the special 2 nd approximation* 
has the smaller reflected error $O\left(|\omega / \xi|^{3}+1 / \xi^{2}\right)$ when compared to the 2nd approximation above. (This is confirmed numerically.) We remark that the conditions in (2.21) are satisfied for the inflow case of the linearized shallow water equations (see the computations in Section 4 using 2nd approximation*) and often should be valid for the (hyperbolic) equations of fluid dynamics. We also remark here that the choice of ${ }_{1} V_{0}(1,0)$ used in $(2.22)$ is different from the one in (2.19), but $V_{0}$ is not unique anyway.

To verify the claim in (2.22) we choose $R$ in the form

$$
R=\left(\begin{array}{ll}
Y & X \\
0 & 0
\end{array}\right)
$$

When we compute $S(A+\delta E) S^{-1}$, a tedious calculation establishes that the terms of order $\delta, \delta^{2}$ appearing in the upper right-hand block of (2.22) and required to vanish have the form,

$$
\begin{array}{ll}
(\text { order } \delta) & -\Lambda_{1} X+X \Lambda_{2}+E_{12}=0 \\
\left(\text { order } \delta^{2}\right) & -E_{11} X+Y\left(-\Lambda_{1} X+E_{12}\right)+\Lambda_{1} Y X+X E_{22}=0
\end{array}
$$

If we choose $X$ as in (2.20) above to satisfy the order $\delta$ equation and then substitute the order $\delta$ equation in the order $\delta^{2}$ equation, we obtain with $Z=Y X$

$$
\left(\text { order } \delta^{2}\right) \quad-Z \Lambda_{2}+\Lambda_{1} Z-E_{11} X+X E_{22}=0 .
$$

Thus, $Z$ is uniquely determined in exactly the same fashion as $X$ above (since $X$ is already known). To recover $Y$, we must determine $Y$ from the relationship

$$
Z=Y X \text {, }
$$

where $Z, X$ are $k \times(m-k)$ matrices and $Y$ is a $k \times k$ matrix. By the hypotheses in (2.21) the $m-k$ columns of $X$ are independent and since $m-k \leqslant k$, we construct $Y$ so that it maps the $(m-k)$ columns of $X$, respectively, onto the $m-k$ columns of $Z$ (thus satisfying (2.27)) and on the orthogonal complement of the subspace of $R^{k}$ spanned by the columns of $X$, choose $Y \equiv 0$.

Remark \#1. The special second approximation yields reflected waves with amplitudes of the same order as the third approximation. The special second approximation utilizes only a first order boundary condition and from a numerical point of view, this approximation is much easier to discretize in a stable fashion than the second order boundary condition required by the third approximation.

Remark \#2. Of course, the special second approximation can be derived whenever it is possible to solve the equation, $Z=Y X$, even though the additional hypotheses from (2.21) are not satisfied.

3. Highly Absorbing Boundary Conditions for the Linearized Shallow Water Equations. We apply the theory developed in Section 2 to the linearized shallow water equations in the half-space $x \geqslant 0$. Thus, we consider a system of the form

$$
\frac{\partial u}{\partial t}=\left(\begin{array}{lll}
a & 0 & c \\
0 & a & 0 \\
c & 0 & a
\end{array}\right) \frac{\partial u}{\partial x}+\left(\begin{array}{lll}
b & 0 & 0 \\
0 & b & c \\
0 & c & b
\end{array}\right) \frac{\partial u}{\partial y}+\left(\begin{array}{rrr}
0 & f & 0 \\
-f & 0 & 0 \\
0 & 0 & 0
\end{array}\right) u
$$


together with the physical restrictions, $0<a^{2}+b^{2}<c^{2}, c>0$. Here for simplicity we assume all matrices in (3.1) are constant. We diagonalize the normal matrix to $x=0$ by the unitary map, $U$, where

$$
U=\left(\begin{array}{ccc}
\frac{1}{\sqrt{2}} & 0 & \frac{1}{\sqrt{2}} \\
0 & 1 & 0 \\
\frac{-1}{\sqrt{2}} & 0 & \frac{1}{\sqrt{2}}
\end{array}\right)
$$

and set $w=U^{-1} u$ to get from (3.1), a new equation for $w$ of the form

$$
\begin{aligned}
\frac{\partial w}{\partial t}= & \left(\begin{array}{ccc}
a-c & 0 & 0 \\
0 & a & 0 \\
0 & 0 & a+c
\end{array}\right) \frac{\partial w}{\partial x}+\left(\begin{array}{ccc}
b & \frac{-c}{\sqrt{2}} & 0 \\
-\frac{c}{\sqrt{2}} & b & \frac{c}{\sqrt{2}} \\
0 & \frac{c}{\sqrt{2}} & b
\end{array}\right) \frac{\partial w}{\partial y} \\
& +\left(\begin{array}{ccc}
0 & \frac{f}{\sqrt{2}} & 0 \\
-\frac{f}{\sqrt{2}} & 0 & -\frac{f}{\sqrt{2}} \\
0 & \frac{f}{\sqrt{2}} & 0
\end{array}\right) w .
\end{aligned}
$$

Writing this equation in the form of (2.3), (3.2) becomes

$$
\begin{aligned}
& \frac{\partial w}{\partial x}=\left(\begin{array}{ccc}
\frac{1}{a-c} & 0 & 0 \\
0 & \frac{1}{a} & 0 \\
0 & 0 & \frac{1}{a+c}
\end{array}\right) \frac{\partial w}{\partial t}+\left(\begin{array}{ccc}
\frac{-b}{a-c} & \frac{c}{(a-c) \sqrt{2}} & 0 \\
\frac{c}{a \sqrt{2}} & \frac{-b}{a} & -\frac{c}{a \sqrt{2}} \\
0 & -\frac{c}{(a+c) \sqrt{2}} & -\frac{b}{(a+b) \sqrt{2}}
\end{array}\right) \frac{\partial w}{\partial y} \\
& +\left(\begin{array}{ccc}
0 & \frac{-f}{(a-c) \sqrt{2}} & 0 \\
\frac{f}{a \sqrt{2}} & 0 & \frac{f}{a \sqrt{2}} \\
0 & \frac{-f}{(a+c) \sqrt{2}} & 0
\end{array}\right) w \text {. }
\end{aligned}
$$

The boundary conditions we choose depend upon whether we have linearized about an inflowing state with $a<0$ or about an outflowing state with $a>0$.

3.A. The Inflow Case. Here we assume $a<0$ so that the normal matrix to the boundary has the form 


$$
\left(\begin{array}{cc}
\Lambda_{1}<0 & 0 \\
0 & \Lambda_{2}>0
\end{array}\right)
$$

where $\Lambda_{1}, \Lambda_{2}$ are given by

$$
\Lambda_{1}=\left(\begin{array}{cc}
\frac{1}{a-c} & 0 \\
0 & \frac{1}{a}
\end{array}\right), \quad \Lambda_{2}=\frac{1}{a+c} .
$$

Following (2.14), we have the first absorbing approximation

1st approximation:

$$
\left.\left(\begin{array}{l}
w_{1} \\
w_{2}
\end{array}\right)\right|_{x=0}=0 .
$$

Following the procedure at the end of Section 2, we choose $X$ to satisfy (2.20) so that $X$ is given by

$$
X=\left(\begin{array}{c}
0 \\
-\frac{a+c}{\sqrt{2}}
\end{array}\right) .
$$

Thus, the hypotheses in (2.21) for the special 2 nd approximation are satisfied. We choose $Z$ to satisfy (2.26) so that $Z$ is given by

$$
Z=\left(\begin{array}{c}
-\frac{(a+c)^{2}}{4} \\
-\frac{b(a+c)}{2 c}((1-\sqrt{2}) a-\sqrt{2} c)
\end{array}\right)
$$

Thus, $Y$ with $Z=Y X$ is given by

$$
Y=\left(\begin{array}{cc}
0 & \frac{\sqrt{2}}{4}(a+c) \\
0 & \frac{\sqrt{2}}{2} \frac{b}{c}((1-\sqrt{2}) a-\sqrt{2} c)
\end{array}\right) .
$$

Thus, $R$ from (2.24) has been computed.

Finally, to calculate the effect to the lower order term corresponding to the Coriolis force on the special second approximation, we need to compute $K_{1}(1,0)$ from the equation in (2.9)-(2.11). Thus, $K_{1}(1,0)$ is given by

$$
K_{1}(1,0)=\left(\begin{array}{c}
0 \\
\frac{(a+c) f}{c \sqrt{2}}
\end{array}\right)
$$


Inserting the values for $R$ in (2.24) obtained from (3.6) and (3.7) and the value of $V_{-1}(1,0)$ determined by $(3.8)$, we obtain the special 2 nd absorbing approximation, 2nd approximation*:

$$
\frac{\partial w_{1}}{\partial t}+\left.\frac{\sqrt{2}}{4}(a+c) \frac{\partial w_{2}}{\partial y}\right|_{x=0}=0
$$

$$
\frac{\partial w_{2}}{\partial t}+\frac{\sqrt{2}}{2} \frac{b}{c}(a-\sqrt{2}(a+c)) \frac{\partial w_{2}}{\partial y}-\frac{a+c}{\sqrt{2}} \frac{\partial w_{3}}{\partial y}+\left.\frac{(a+c) f}{c \sqrt{2}} w_{3}\right|_{x=0}=0
$$

It is worthwhile mentioning that the $1 \frac{1}{2}$ absorbing approximation is given by

$$
\left.\frac{\partial w_{1}}{\partial t}\right|_{x=0}=0, \quad \frac{\partial w_{2}}{\partial t}+\left.\frac{a+c}{c \sqrt{2}} f w_{3}\right|_{x=0}=0 .
$$

3.B. The Outflow Case. Here we assume $a>0$, so that

$$
0>\Lambda_{1}=\frac{1}{a-c}, \quad 0<\Lambda_{2}=\left(\begin{array}{cc}
\frac{1}{a} & 0 \\
0 & \frac{1}{a+c}
\end{array}\right) \text {. }
$$

In this case the first absorbing approximation is

$$
\left.w_{1}\right|_{x=0}=0 \text {. }
$$

Following the computations as in the inflow case, we calculate that

$$
X=\left(\frac{a}{\sqrt{2}}, 0\right), \quad K_{1}=\left(-\frac{a f}{c \sqrt{2}}, 0\right) .
$$

On the other hand, one calculates that $Z=\left(b_{1}, b_{2}\right)$ with $b_{2} \neq 0$ so it is impossible to find a $1 \times 1$ matrix $Y$ with $Z=Y X$ so the special second approximation is not applicable. Thus, following (2.15) we have the second absorbing approximation

2nd approximation:

$$
\frac{\partial w_{1}}{\partial t}+\frac{a}{\sqrt{2}} \frac{\partial w_{2}}{\partial y}-\left.\frac{a}{c \sqrt{2}} f w_{2}\right|_{x=0}=0
$$

\section{Difference Approximations for Absorbing Boundary Conditions-Numerical} Experiments.

A. The Scalar Wave Equation. Here we discuss the difference approximation of the scalar wave equation and the highly absorbing boundary conditions from Section 1 and then present the results of numerical experiments which illustrate their effectiveness. We look at the scalar wave equation from (1.1) in the half-space, $x \geqslant 0$, and approximate the solution $w(t, x, y)$ on the grid $\left\{\left(t^{n}, x_{j}, y_{k}\right)\right\}$ by the mesh function $w_{j, k}^{n}\left(t^{n}=n \Delta t, x_{j}=j \Delta x-\Delta x / 2, y_{k}=k \Delta y ; n=0,1, \ldots, j=0,1, \ldots, k=\right.$ $\ldots,-1,0,1, \ldots)$. We use the notation $D_{+}, D_{-}$, and $D_{0}=1 / 2\left(D_{+}+D_{-}\right)$to denote the forward, backward, and centered divided differences, respectively. The equation 
in (1.1) is approximated by the simple explicit formula

$$
\begin{aligned}
& \left(D_{+}^{t} D_{-}^{t}-D_{+}^{x} D_{-}^{x}-D_{+}^{y} D_{-}^{y}\right) w_{j, k}^{n}=0, \\
& \quad n=1,2, \ldots, j=1,2, \ldots, k=\ldots,-1,0,1, \ldots .
\end{aligned}
$$

The values of $w_{j, k}^{0}$ and $w_{j, k}^{1}$ are given initially.

We discretize the first approximation from (1.9) by

$$
D_{+}^{x}\left(w_{0, k}^{n}+w_{0, k}^{n+1}\right)-D_{+}^{t}\left(w_{0, k}^{n}+w_{1, k}^{n}\right)=0
$$

while we use the following difference approximation for the second highly absorbing boundary condition from (1.10):

$$
D_{0}^{t} D_{+}^{x} w_{0, k}^{n}-1 / 2 D_{+}^{t} D_{-}^{t}\left(w_{0, k}^{n}+w_{1, k}^{n}\right)+1 / 4 D_{+}^{y} D_{-}^{y}\left(w_{0, k}^{n-1}+w_{1, k}^{n+1}\right)=0
$$

For the numerical experiments presented below, we have discretized the Dirichlet and Neumann problems in the obvious fashion using $w_{0, k}^{n+1}=0$ and $D_{-x}^{x} w_{0, k}^{n+1}=0$, respectively. It is easy to check that (4.1) coupled to (4.2) or (4.3) gives an explicit scheme with a local truncation error of second order in all variables. (This is also obvious for the approximations for the Dirichlet and Neumann boundary conditions which we have used.) Tests with initial data containing high frequencies gave numerical evidence of the stability of these approximations to the mixed initial boundary value problems. The boundary conditions can, of course, be coupled to any type of interior discretization.

In Figure 1 we display the results from a series of experiments for the scalar wave equation. The first Figure (1a) shows the initial values $w_{j, k}^{0}$. Each vertical trace corresponds to a vector $w_{j, k}^{n}$ with $n$ and $j$ fixed; different traces correspond to different $j$ 's. One hundred percent black is $w \equiv 1$ and $30 \%$ black is $w \equiv 0$ on these traces. The initial values $w_{j, k}^{1}$ are chosen using Taylor's theorem so that the circular wave form expands with time. For all experiments we have used the discrete Neumann boundary condition on the upper, lower, and right-hand walls. We will change our boundary condition only on the left wall corresponding to $x=0$. In Figure (1b) we have the plot after 50 time steps using the perfectly reflecting Neumann boundary condition on $x=0$. Figure (1c) displays the same solution after 100 time steps. (We used $\Delta x$ $=\Delta y=2.5 \Delta t, 0 \leqslant k \leqslant 50,0 \leqslant j \leqslant 100$.) Figures (1d), (1e), and (1f) all describe various solutions of (4.1) after 100 time steps. The discrete Dirichlet boundary condition was used on the wall, $x=0$, in Figure (1d); waves are totally reflected. Figure (1e) represents the solution of (4.1) using the absorbing first approximation from (4.2); there is a weak reflected wave with amplitude in the $L^{2}$ norm about $5 \%$ of the incident amplitude. Figure (1f) represents the solution of (4.1) using the second absorbing approximation from (4.3); the weak negative reflection is not observed here. In fact, the reflected wave has amplitude in the $L^{2}$ norm smaller than $1 \%$ of the amplitude of the incident wave. 


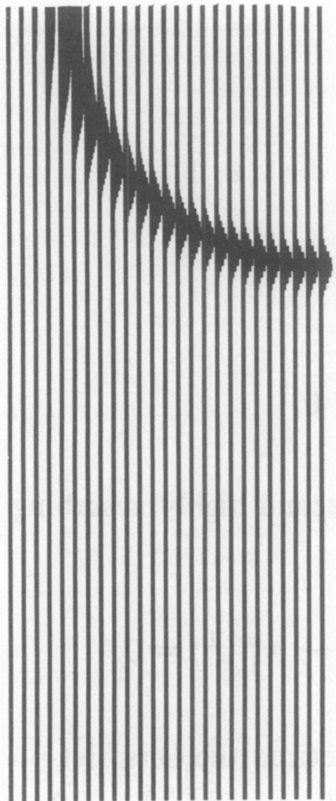

FIGURE 1A

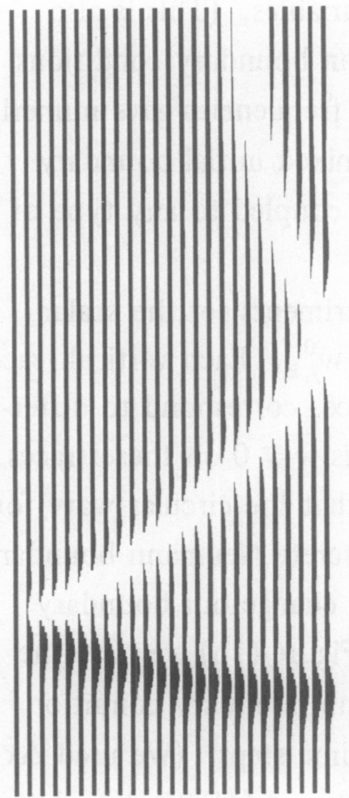

FiguRE 1D

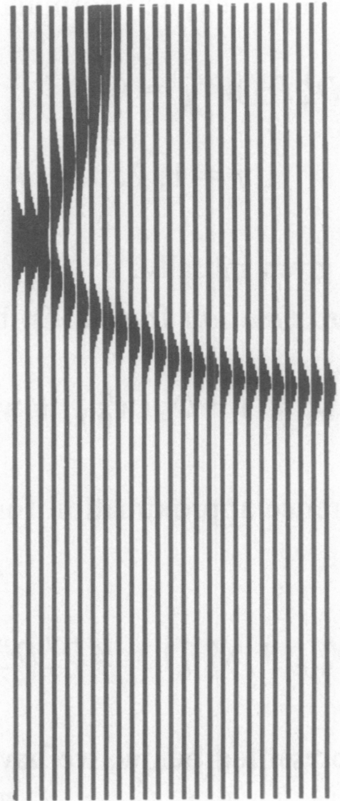

Figure 1B

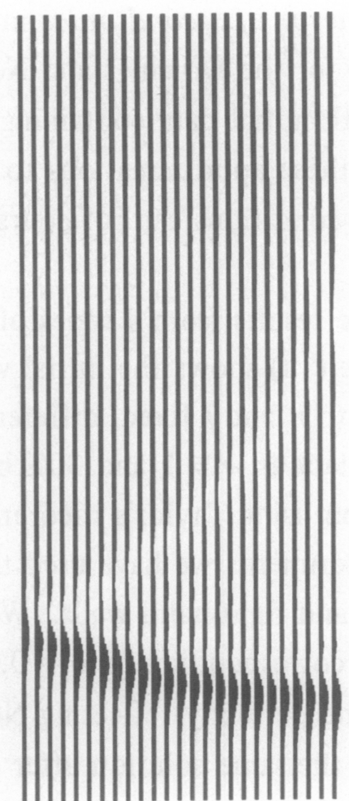

FIGURE 1E

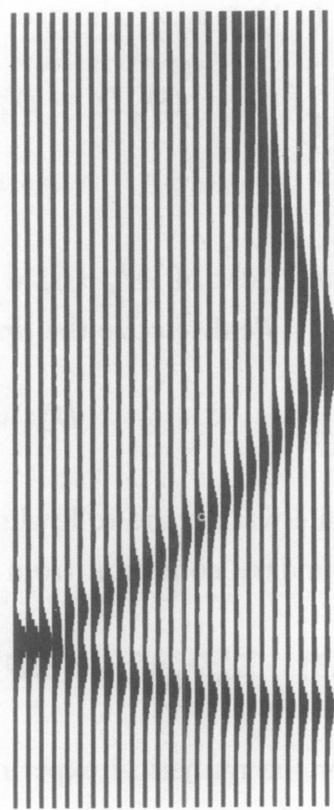

Figure 1C

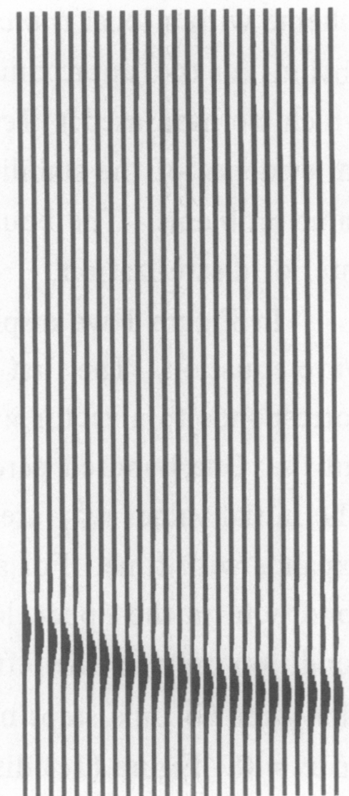

FIGURE 1F

B. The Shallow Water Equation. As the last example, we will approximate the solution to the linearized shallow water equation from (3.1), (3.2) when $f=0$. We have chosen to study the inflow case when $a<0, b>0$, and $c>0$. The first and second (order) absorbing boundary conditions are then given by (3.5) and (3.9), respectively. It is outside of the scope of this paper to make a thorough study of possible difference approximations to that mixed initial boundary value problem. We will 


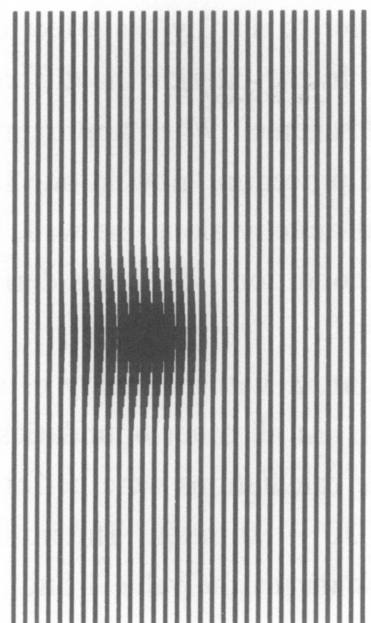

Figure 2A

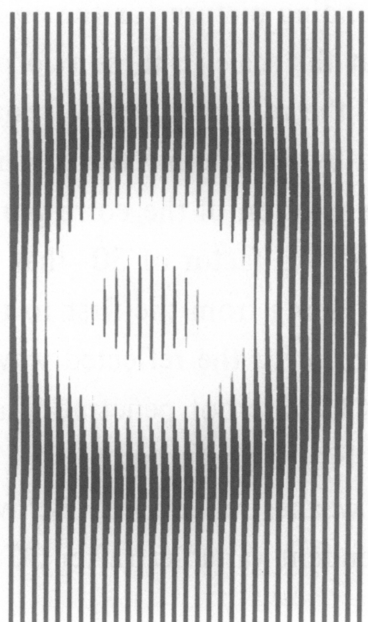

Figure 2C

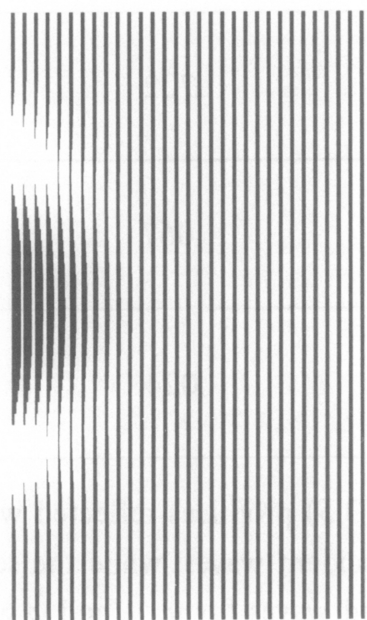

Figure 2E

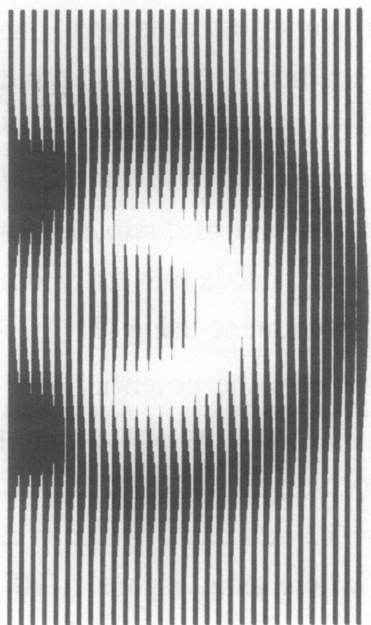

Figure 2B

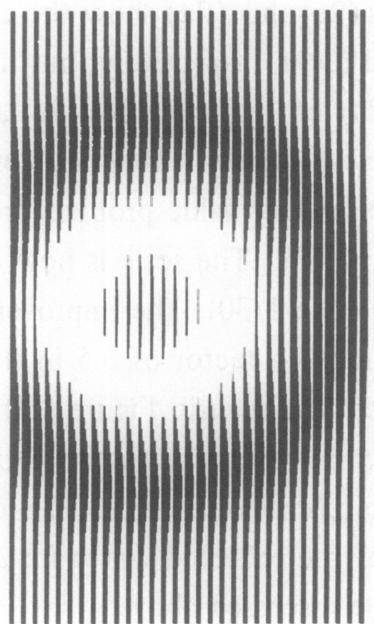

Figure 2D

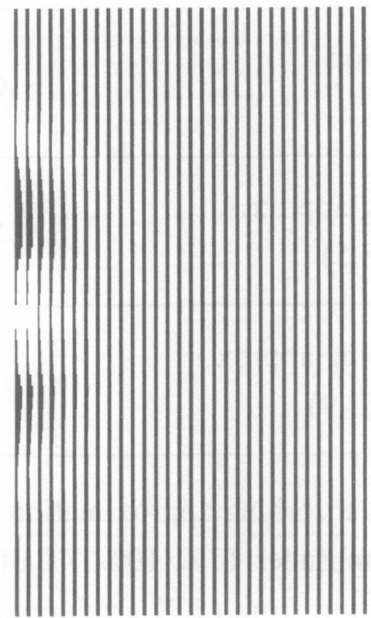

Figure 2F 
only report on some test runs performed in order to display the effect of the analytical absorbing conditions from (3.5) and (3.9). In the interior the differential equation was approximated by a standard Lax-Wendroff scheme. When approximating the boundary condition in (3.9), the Lax-Wendroff procedure, now in one space dimension and with added numerical dissipation, was used directly along the wall, $x=0$. The difference equation needs one extra boundary condition. That was derived by extrapolating the $w_{3}$-component using $\left(D_{+}^{x}\right)^{3}\left(w_{3}\right)_{0, j}^{n}=0$.

Figure 2 shows the potential field or height, $\varphi$, with boundary of interest to the left at $x=0$ ( $\varphi$ is the third vector component). At all other boundaries we used the first order absorbing condition from (3.5). The calculations were performed with $a=$ $-1, b=2, c=12$ and $25 \Delta t=\Delta x=\Delta y$ on a $30 \times 50$ mesh. The initial $\varphi$ is displayed in Figure (2a) where as in Figure 1, 100\% black means the value 1 and 30\% stands for zero. The first two components, the velocities $u$ and $v$ were taken as zero initially. In Figure (2b), $\varphi$ is given after 30 time steps using the reflecting condition $u=v=0$ at $x=0$. Figures $(2 \mathrm{c})$ and $(2 \mathrm{~d})$ show the corresponding picture for the absorbing conditions (3.5) and (3.9), respectively; but note that the scale in these two plots is multiplied by a factor of 3 . In Figures (2e) and (2f) the reflected waves from (2c) and (2d) are displayed, respectively. They are computed by subtracting the solution of this mixed initial boundary value problem from the solution of the corresponding pure initial value problem. The scale is now changed by a factor of 30 -that is, $100 \%$ black stands for the value $1 / 30$. The improvement in going from the first to the second $a b$ sorbing condition is a factor of 3.5 in the $L_{2}$-norm for the reflected wave. Furthermore, the reflections seen in Figure (2f) using (3.9) are not penetrating as deeply into the region of computation as the reflections in Figure (2e) using (3.5). Thus, as expected the approximation from (3.9) produces weaker reflections. Below, we display the $L_{2}$-norm of the reflected waves for all dependent variables (after 30 time steps) for the example discussed above:

Boundary condition at $x=0 \quad \| u$ reflected $\left\|_{2} \quad\right\| v$ reflected $\left\|_{2} \quad\right\| \varphi$ reflected $\|_{2}$ perfect reflection $u=v=0$ .621 .702 .695

First absorbing approx. from (3.5) .103 .067 .053

Second absorbing approx. from (3.9)

Thus, the first absorbing approximation from (3.5) produces reflected waves for $u, v$, and $\varphi$ with amplitudes $17 \%, 10 \%, 7 \%$ of the incident waves, while the second absorbing approximation from (3.9) produces reflected waves for $u, v$, and $\varphi$ with amplitudes $4 \%, 3.3 \%$, and $2 \%$ of the incident waves, respectively. 
Department of Computer Science

Uppsala University

Uppsala, Sweden

Department of Mathematics

University of California

Los Angeles, California 90024

1. H.-O. KREISS, "Initial boundary value problems for hyperbolic systems," Comm. Pure Appl. Math., v. 23, 1970, pp. 277-298.

2. A. MAJDA \& S. OSHER, "Reflection of singularities at the boundary," Comm. Pure Appl. Math., v. 28, 1975, pp. 479-499.

3. L. NIRENBERG, Lectures on Linear Partial Differential Equations, C.B.M.S. Regional Conf. Ser. in Math., no. 17, Amer. Math. Soc., Providence, R. I., 1973.

4. M. E. TAYLOR, "Reflection of singularities of solutions to systems of differential equations," Comm. Pure Appl. Math., v. 28, 1975, pp. 457-478.

5. F. J. MASSEY III \& J. B. RAUCH, "Differentiability of solutions to hyperbolic initialboundary value problems," Trans. Amer. Math. Soc., v. 189, 1974, pp. 303-318. MR 49 \#5582.

6. DAVID M. BOORE, "Finite difference methods for seismic wave propagation in heterogeneous materials," Methods of Comp. Physics (Seismology), v. 11, 1972, pp. 1-37.

7. K. R. KELLY, R. M. ALFORD, S. TREITEL \& R. W. WARD, Applications of Finite Difference Methods to Exploration Seismology, Proc. Roy. Irish Acad. Conf. on Numerical Analysis, Academic Press, London and New York, 1974, pp. 57-76.

8. P. J. ROACHE, Computational Fluid Dynamics, Hermosa Press, Albuquerque, N. M., 1972.

9. T. ELVIUS \& A. SUNDSTRÖM, "Computationally efficient schemes and boundary conditions for a fine mesh barotropic model based on the shallow water equations," Tellus, v. 25, 1973, pp. $132-156$.

10. E. L. LINDMAN, "Free space boundary conditions for the time dependent wave equation," J. Computational Phys., v. 18, 1975, pp. 66-78.

11. I. ORLANSKI, "A simple boundary condition for unbounded hyperbolic flows," $J$. Computational Phys., v. 21, 1976, pp. 251-269.

12. M. E. HANSON \& A. G. PETSCHEK, "A boundary condition for sufficiently reducing boundary reflection with a Lagrangian mesh," J. Computational Phys., v. 21, 1976, pp. 333-339.

13. W. D. SMITH, "A nonreflecting plane boundary for wave propagation problems," $J$. Computational Phys., v. 15, 1974, pp. 492-503. 